\title{
Abwertung der «Praxis»-CMEs durch die Schweizerische Gesellschaft für Allgemeine Innere Medizin
}

\author{
Edouard Battegay ${ }^{1,2,3}$ und Markus Schneemann ${ }^{4}$ \\ ${ }^{1}$ International Center for Multimorbidity and Complexity in Medicine (ICMC), Universität Zürich \\ ${ }^{2}$ Universitätsspital Basel (Klinik für Psychosomatik) \\ ${ }^{3}$ Merian Iselin Klinik, Basel \\ ${ }^{4}$ Medizinische Klinik, Kantonsspital Schaffhausen
}

Liebe Lesende

Lange haben wir argumentiert, aber trotz letztlich nicht nachvollziehbarer Argumente seitens der Schweizerischen Gesellschaft für Allgemeine Innere Medizin (SGAIM) vorderhand den Kürzeren gezogen: Ab 2022 hat die SGAIM, die Fachgesellschaft der meisten von uns, die Creditpunkte für CME-Beiträge in der «Praxis» halbiert. Ab sofort müssen für die Erlangung eines einzigen FortbildungsCreditpunkts zwei CME-Beiträge der «Praxis» bearbeitet und die Fragen dazu zu $60 \%$ korrekt beantwortet werden.

Das Argument der SGAIM: Für die Bearbeitung eines CME-Beitrags in der «Praxis» sowie der (korrekten) Beantwortung der Fragen braucht es weniger als 50 Minuten (in Worten: FÜNFZIG Minuten). Wir haben das intern mit Probelesen geprüft und wir haben Sie gebeten, uns Ihre persönlichen Bearbeitungszeiten mitzuteilen - wir kommen auf längere Bearbeitungszeiten. Das ist aber sicherlich debattierbar, denn empirische Forschung zeigt: Lesezeiten variieren sehr.

Unsere Argumentation bezüglich Lesezeit hat aber eine Basis in Publikationen. Es lohnt sich, hier die Aussage von Hans Asbjørn Holm, dem damaligen Vizegeneralsekretär der norwegischen Ärztevereinigung, im British Medical Journal des Jahres 2000 zu zitieren [1]: «If we take into account the pivotal role that reading has in a doctor's continuous learning then reading should be generously honoured, allowing doctors to meet at least half of any set annual standard of credit points by reporting their reading and its perceived influence on their practice.»

Erstaunlicherweise entspricht Lesen aber auch einem generellen Trend vor allem jüngerer Ärztinnen und Ärzte, sich fortzubilden. Aus Gründen der Dienstorganisation oder der familiären Situation bewegen sich Jüngere oft kaum an Kongresse, die weniger auf Ihre Bedürfnisse eingehen, sondern bilden sich eher am Laptop oder beim Lesen weiter. Dieser Trend hat sich unter Corona-Bedingungen mit Sicherheit allseits beschleunigt und bei allen Altersgruppen verstärkt.
Zudem haben wir gegenüber der SGAIM ähnlich wie Holm in seinem ausgezeichneten und wirklich lesenswerten Editorial im BMJ argumentiert, dass die Bearbeitungsdauer nicht DAS Argument für die Beurteilung der Qualität eines CME-Beitrags sein kann. Wie bereits erwähnt, wird in verschiedenen Publikationen gezeigt, dass die Bearbeitungsdauer eines Beitrags sehr variieren kann UND dass zwischen der Bearbeitungszeit und der Zeichenzahl (als Parameter für den Artikelumfang) nur eine schwachlineare Korrelation besteht [2,3]. Die Autorinnen und Autoren kommen zum Schluss, dass der Umfang eines Artikels bzw. die reine Lesezeit von untergeordneter Bedeutung ist. Die SGAIM lässt dies unbeeindruckt.

Auch hoben wir gegenüber der SGAIM wiederholt hervor, dass die «Praxis» eine unabhängige Zeitschrift ist, in der keine gesponserten Artikel erscheinen - und doch wird uns das Erlangen von Credits in diesem Umfeld zunehmend schwergemacht. Uns fallen hingegen immer wieder von der SGAIM akkreditierte Beiträge auf, bei denen die Unterstützung durch die Industrie eindeutig ist, was bei einer seriösen Fachgesellschaft nicht der Fall sein darf [4]. Bitte achten Sie hier auf die Creditvergabe durch die SGAIM! Sehr interessant, finden wir.

Wir weisen an dieser Stelle auch darauf hin, dass unsere CME-Artikel in Niedersachsen - wir haben dies verlagsintern geprüft - mehr Creditpunkte erhalten würden als von der SGAIM zugestanden. Es ist denkbar, dass hier eine Ungleichbehandlung von Ärztinnen und Ärzten entstehen könnte, wenn diese ihre Fortbildung auf der Basis unserer CMEs nicht hierzulande, sondern anderswo und für Deutschland akkreditieren liessen.

Die gute Qualität der Beiträge wurde uns von der SGAIM wiederholt bestätigt... dennoch: Dem negativen Entscheid haben wir im Oktober umgehend und in wiederholtem Mailwechsel widersprochen, aber bis zur Drucklegung dieses Beitrags Mitte Dezember lag uns noch keine Rückmeldung auf unsere Argumente vor.

Die «Praxis» ist nicht die einzige Schweizer Zeitschrift, die von dieser kaum nachvollziehbaren Entscheidungspolitik betroffen ist. Nun sind Sie, die Leserinnen und Le- 
ser, gefragt. Wenden Sie sich an die SGAIM, rufen Sie an und schreiben Sie: Fort- und Weiterbildung in der Schweiz muss auf vielen Wegen möglich sein. Nicht nur in Zeiten von COVID-19.

Viele Autorinnen und Autoren haben in den vergangenen Jahren immens viel Zeit und Energie in die CMEs gesteckt, um Kolleginnen und Kollegen Fortbildung auf diesem Weg zu ermöglichen - mit nicht mal einem Creditpunkt pro Beitrag ist es schwer, die Motivation beizubehalten, einen Beitrag zu verfassen. Es wird 2022 nicht in jeder Ausgabe der «Praxis» die Möglichkeit bestehen, Fortbildungs-Credits zu erlangen, so in der vorliegenden Ausgabe.

Aber wir freuen uns, wenn Sie trotz der reduzierten Ausbeute auch weiterhin die CMEs bearbeiten. Wenn Sie Ihre Kolleginnen und Kollegen durch das Verfassen von CME-Beiträgen unterstützen wollen, senden Sie Vorschläge an die Redaktion.

\section{Edouard Battegay und Markus Schneemann}

PS: Mit dem Read-o-Meter (https://niram.org/read/) geschätzte Lesezeit dieses Editorials (mit Unterschriften, aber ohne PS): 3 Minuten, 16 Sekunden

\section{Bibliografie}

1. Holm HA. Should doctors get CME points for reading. BMJ 2000; 320(7232):394-395. https://www.ncbi.nlm.nih.gov/pmc/ articles/PMC1117529/; letzter Zugriff: 02.12.2021.

2. Schäfer $S$, Schäfer $C$, Lösche $P$, et al. Determinanten für die Bearbeitungszeit von zertifizierter Fortbildung in Printmedien. Med Klinik. 2008;103:230-240.

3. Weiner $\mathrm{C}$, Vaugh $\mathrm{K}$. The effects of reading speed and retrieval practice on reading comprehension. Nysa 2019;2:2-5.

4. Spithoff S. Industry involvement in continuing medical education. Can Fam Physician. 2014;60:694-696.

\section{Prof. Dr. Edouard Battegay}

Leiter International Center for Multimorbidity and Complexity in Medicine (ICMC), Universität Zürich Universitätsspital Basel

Merian Iselin Klinik

edouard.battegay@uzh.ch

PD Dr. med. Markus Schneemann

Chefarzt Innere Medizin

Kantonsspital

Spitäler Schaffhausen

8208 Schaffhausen

Markus.Schneemann@spitaeler-sh.ch 